\title{
INFLUENCE OF TOOL BALANCING IN HIGH SPEED MACHINING
}

\author{
KLAUDIA BAŠOVSKÁ \\ Faculty of Material Science and Technology in Trnava/Institute of Production Technologies, Department of \\ Machining, Assembly and Forming, Slovak University of Technology in Bratislava, Trnava, Slovakia, \\ klaudia.basovska@stuba.sk \\ JOZEF PETERKA \\ Faculty of Material Science and Technology in Trnava/Institute of Production Technologies, Department of \\ Machining, Assembly and Forming, Slovak University of Technology in Bratislava, Trnava, Slovakia, \\ jozef.peterka@stuba.sk
}

\begin{abstract}
The high speed machining (HSM) is now considered as one of the key manufacturing technologies for higher throughput and productivity. HSM used higher rotational speed of the spindle (40,000 min-1 and higher). With increasing high speed spindle rotations raises a number of dynamic forces. Even a small mass unbalance in the spindle and tooling generates tool vibration. Tool vibration shortens tool life and lowers the quality of the machined surface. It is necessary to minimize this vibration by balancing tool and tool holder. The balancing process improves the mass distribution of a cutting tool and its holder, allowing the combination of the two to rotate with the minimum amount of unbalanced centrifugal forces. Machining with balanced tool will provide better surface quality, accuracy and less tool and machine wear. In this study is focused on unbalance cutting tools, definitions, balancing techniques, sources, effects, processes and machineries. The aim of this article was to examine the relationship between unbalance and tool holders used in high speed metalworking machine tools.
\end{abstract}

Keywords: unbalance, balancing, dynamic unbalance

\section{Introduction}

High Speed Machining enables manufacturers to shorten machining times and to achieve a higher surface quality. The result is tighter-tolerance parts with improved surface finishes. However, at high speeds, unbalance in the tool and tool holder can cause significant damage to the spindle bearings and to the spindles itself.

At speeds higher than 8,000 rpm it is generally recommended to balance the tool and tool holder as an assembly. Despite the relatively small mass of this assembly compared to the spindle, at these higher speeds small amounts of unbalance create large amounts of centrifugal force (Moak 2001). It is this force which creates the vibration which in turn can damage the spindle and/or bearings and perform other undesirable effects such as chatter, poor surface finish, high tool wear, etc. (McMillan 2004).

\section{Unbalance}

Unbalance is a condition which exists in a rotor when vibration force or motion is imparted to its bearings as a result of centrifugal forces (ISO1940/1).

Mass unbalance occurs in a rotating machine when the mass of the rotor is unevenly around the center of rotation. When this happens the center of mass does not correspond with the center of rotation (Figure1)(Commtest 2005). 


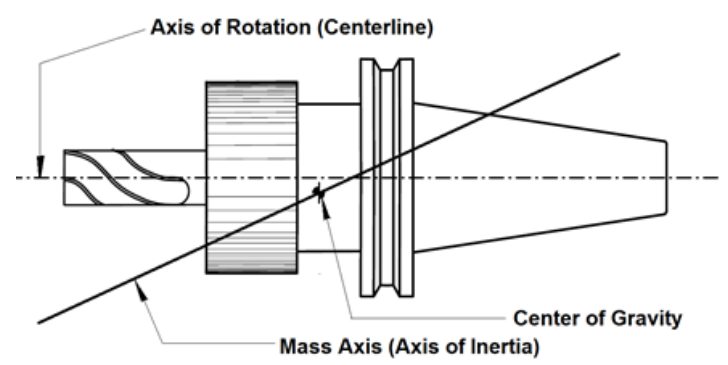

Unbalance can be of three basic types: static, couple and dynamic (Figure 2). Static unbalance is defined as an unbalance where the center of rotation is displaced parallel to the geometric center of the rotating element while dynamic exhibits different amplitudes of vibration at each end of the rotating element (McMillan 2004).

Figure 2 Types of unbalance a) static b) couple c) dynamic(Layne 2007)

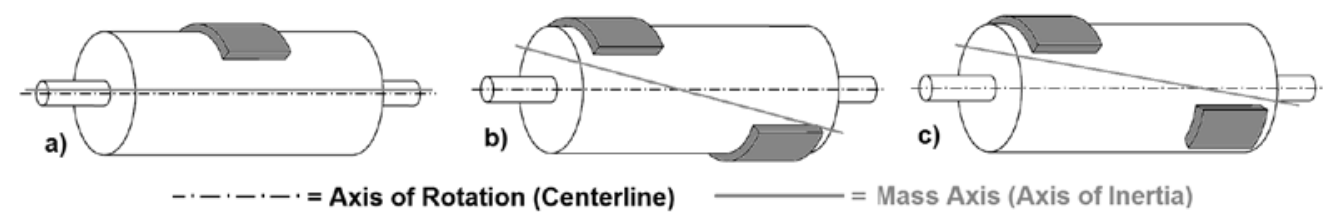

\subsection{The Effects of Unbalance}

The detrimental effects of tool and tool holder unbalance can be divided into following categories.

Effects on the work piece - the primary result of the tool holder unbalance on the work piece is that of chatter, or ripples in the metal surface caused by the movement of the cutting tool. High frequency chatter can also result in a poor surface finish. The inability to hold close tolerances, which means more scrapped parts, presents the second effect of unbalance on the work piece.

Effects on the machine - the centrifugal forces will cause tremendous internal stress in the spindle, normally resulting in premature spindle bearing failure. Vibration is energy, and if the energy cannot get away from the spindle via the ways, then the only other path is through the work piece via the tool holder and cutting tool. This usually means a chatter and loss of tool life.

Effects on the cutting tool - as briefly mentioned above, reduced cutting tool life is another negative effect of the tool holder unbalance. Recent research by several major tool holder manufacturers has indicated that tool life can be improved by as much as $50 \%$ by using balanced tool holders (Layne 2007).

\subsection{Causes of Unbalance}

The researches indicated that there are two types of unbalance sources in high speed tool holders namely Controllable (Fixed) and Uncontrollable (Variable) sources (McMillan 2004).

Figure 3 a) Controllable (Fixed) sources, b) Uncontrollable (Variable) Sources(Layne 2007)

a)

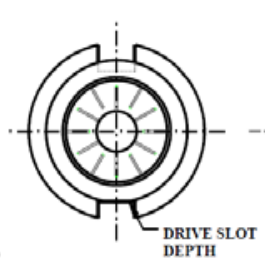

b)

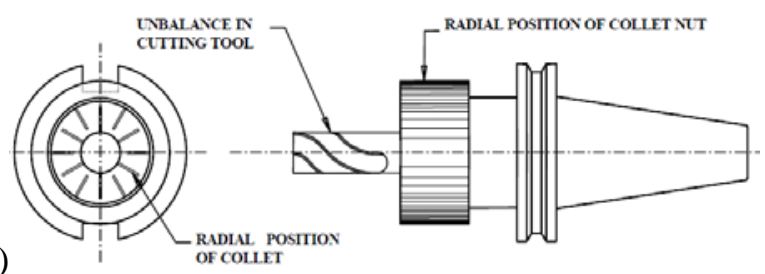

All of Controllable sources of unbalance (drive slot, unground surfaces, retention knob and any other geometric characteristics) can be eliminated by either careful design or balancing of the tool holder by the manufacturer. All Tool holder used in High Speed Machining should be two plane balanced by the tool holder manufacturer. This will insure that all of the Fixed Sources of unbalance have been removed from the tool holder (Figure 3a). 
Uncontrollable sources of unbalance (collet position, collet nut position, cutting tool, set screws) change every time the cutting tool is changed or the collet is loosened and re-clamped. These sources of unbalance usually are located in one part of the tool holder - at the cutting tool end(Layne 2007). Because they are usually cantered around the cutting tool area, in most cases the Variable Sources can be eliminated with single-plane balancing (Figure 3b).

\section{Methods}

Unbalance is present to some extent in all rotating equipment, and has been found to be the most common source of vibration encountered. Field balancing of rotating equipment depends on identifying the type of unbalance and then applying the correct balancing procedure. Single plane balancing is only successful in conditions where the unbalance is pure static. Other forms of unbalance will require two or more planes of correction.

If the tool holder is relatively short in length, the unbalance sources can be expected to produce a single plane unbalance. If, however the tool holder has a length which is more than two times the diameter at the gage line, then two-plane balancing may be necessary.

In general, the unbalance must be either compensated for in the same plane in which it occurs, or resolved into two or more corrective planes to prevent the formation of couple forces(Grim 2008). To prevent the formation of a couple forces, the correction must act in the same plane as the unbalance, or resolve. That is, the sum of the forces and the sum of the moments must equal zero. A moment is a force times the distance from a reference point(Kováč 2011).

Balancing in one plane (static) - compensation for the static portion of an unbalance

- The centre of gravity of a rotor is brought back to the axis of rotation

- The couple unbalance of dynamic unbalance remains unchanged.

Balancing in two planes (dynamic) - complete compensation for unbalance (static and couple)

- In principle, any balancing planes can be selected (Haimer 2009)

\subsection{Methods of Correction}

The Fixed Sources of unbalance can be easily removed from the tool holder by drilling or grinding to remove material and balance the tool holder body. This is done by the tool holder manufacturer as a part of the tool holder manufacturing process. The Variable Sources, however, can only be removed after the tool and tool holder are in their final condition, ready to go into the Milling Machine.

Several Tool holder Manufacturers have developed systems for balancing their tool holders to remove the Variable Sources of unbalance after the:

Axial Tapped Holes - one tool holder manufacturer has created a balancing system for tool holders which consist of 8 to 12 tapped holes which are axially positioned in the tool holder. These tapped holes are used to add set screws and other small balance weights such as balls or rods to offset the unbalance in the tool holder.

Radial Tapped Holes- Tooling Innovations, the creator of the heat shrink tool holder known as the "Shrinker", utilizes a system where they drill and tap several equally spaced holes radially into the tool holder.

Balancing Rings - severaltool holder manufacturers have developed systems which use two rotating rings which are integrated into the body of the tool holder. Both rings have an equal unbalance.

Boring Bars - for boring bars, one company utilizes a system with a built in micrometres is used to set the cutting tool on the boring bar. As the cutting tool is advanced outward radially, an internal counterweight moves off-centre in the opposite direction, keeping the tool in balance at all radial settings (Layne 2007).

Figure 4 a) Axial Tapped Holes, b) Radial Tapped Holes, c) Balancing Rings (Layne 2007)
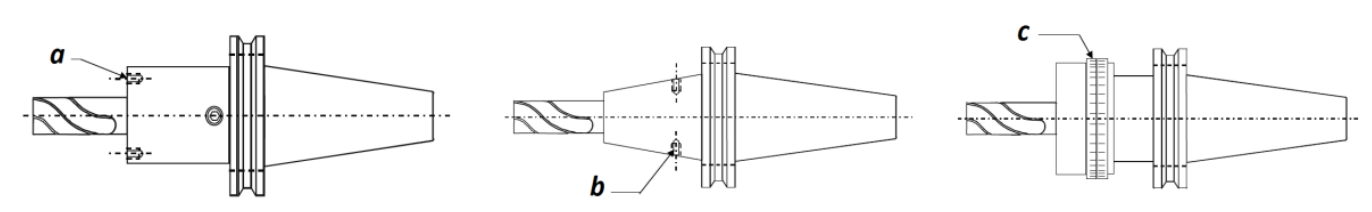


\subsection{Balancing quality}

The principles for measuring unbalance and for balancing are defined in DIN ISO 1940-1. The accuracy of balancing is expressed as balancing quality grade $G$. The balancing quality grade is only ever valid for one particular rotation speed of the rotor (Dingding 2012). The permissible residual unbalance is calculated from the balancing quality grade, the rotation speed and the weight of the rotor. Instead using $e$ (specific unbalance)or $U$ (residual unbalance)to define the balancing quality, it is possible to use $G$ and n from the norm ISO 1940 (ISO 1940/1). The relation between $G$ and $n(\mathrm{rpm})$ in regard to $e$ is shown on the chart taken from the norm ISO 1940.

Figure 5 Unbalance (Seco 2012)

Residual unbalance $U$ :

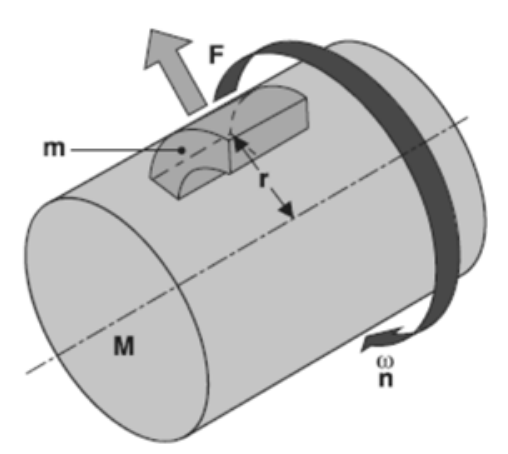

$U=m \cdot r$

$U[\mathrm{~g} . \mathrm{mm}]$ is the product of the unbalance mass $m[\mathrm{~g}]$ and its distance to the rotational axis $r$ [mm]. Value and direction of the residual unbalance $U$ are measured using a balancing machine. Residual unbalance $U$ causes a centrifugal force $F[N]$ to act on the rotating parts. This force will have, for example, a negative effect on the useful life of the spindle bearings.

$F=10^{-6} \cdot U \cdot \omega^{2}$

Balancing is the process which improves the distribution of the mass of a solid piece, in order to reduce $U$ and its effect $F$ to an acceptable level.

Specific unbalance $e$ :

$e=\frac{U}{M}$

$e$ is also called unbalance eccentricity: e $[\mathrm{g} . \mathrm{mm}] /[\mathrm{kg}]$ or $[\mu \mathrm{m}]$ is the distance to which the centre of gravity is offset in regard to the holder's rotational axis. Balancing reduces $e$, in other words, brings back the centre of gravity as near as possible to the axis of rotation (Seco 2012).

$G=\frac{e}{1000} \cdot \omega G=\frac{U}{1000 \cdot M} \cdot \frac{2 \pi n}{60}$

Where: $\mathrm{G}=$ balance quality in $\mathrm{mm} / \mathrm{s}$

$\mathrm{e}=$ specific unbalance in $\mathrm{g} . \mathrm{mm} / \mathrm{kg}$ or $\mu \mathrm{m}$

$\omega=$ speed in $\mathrm{rad} / \mathrm{s}$

$\mathrm{U}=$ residual unbalance, $(\mathrm{U}=\mathrm{e} \times \mathrm{M})$ in g.mm

$\mathrm{M}=$ mass of tool holder in $\mathrm{kg}$

$\mathrm{n}=\mathrm{rpm}$

$\mathrm{m}=$ unbalance mass in $\mathrm{g}$

$\mathrm{r}=$ distance between the unbalance mass and rotational axis in $\mathrm{mm}$

Balancing quality $G$ :

Norm ISO 1940 has established $G$ as the unit of measurement for the balance quality of rotating parts. $G$ is the tangential speed of the centre of gravity in reference to the rotational axis (SandvikCoromant 2012) .G is depending on $n, M$, $U$ : It is not possible to define $G$ without referring to the rotational speed. For toolholders it is preferable to use e and $U$ to define their balancing quality. Graph 1(ISO 1940) showing the relation between $G$ and $n$ in regard to $e$. 


\section{Graph 1. Maximum permissibleresidualunbalance and specificunbalance (Seco 2012)}

ISO 1940

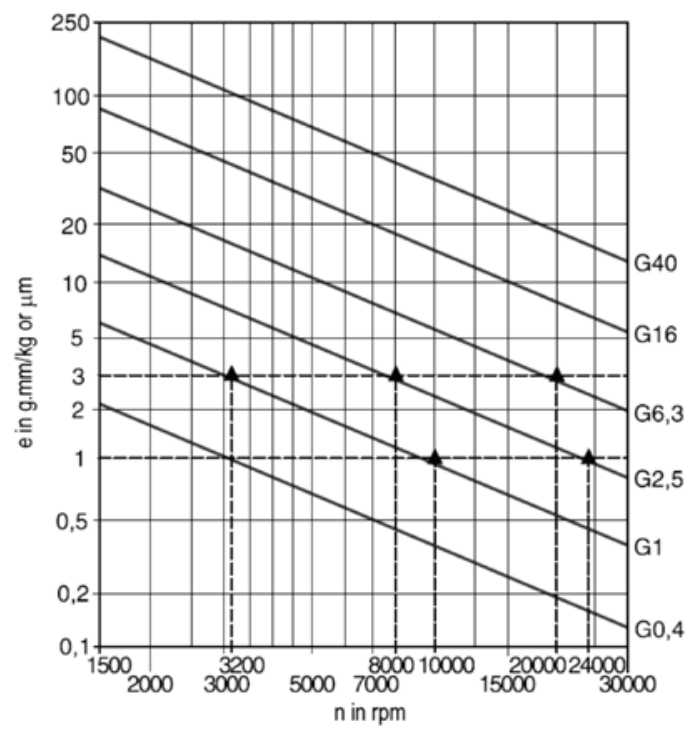

\section{Results}

DIN ISO 1940 assessed tool as a rigid body and by balance quality grade provides unbalance. This method does not take into account the cyclic stress machine tool, which arises as a result of unbalance. It also does not reflect the quality requirements of the surface. If the standard used for light tool at high speed, sets unrealistic, immeasurable and irreproducible values .

For example: specific unbalance $e$ at $25000 \mathrm{rpm}$ and the desired degree of balance $G$ 2,5 is $1 \mathrm{gmm} / \mathrm{kg}$ to the tools of $1 \mathrm{~kg}$ is the eccentricity of the center of gravity $1 \mu \mathrm{m}$ and at even lighter tools at even higher speeds leads to even smaller values .

Furthermore, the standard does not consider the exchange of the cutting tools. Due to inaccuracies clamping constitutes the radial deviation of the HSK clamping $2 \div 4 \mu \mathrm{m}$, there is a change in the balance each time. Another factor limiting balance options are the dimensional tolerances of the individual elements tool assembly. Firstly the inaccuracies resulting from the clamping, which limit the feasibility and reproducibility of the balance quality grade of the whole system spindle / chuck / tool (Borovan 2008).

Therefore, based on existing standards DIN ISO 1940 developed procedures that can help you establish new requirements for rotating tool systems taking into account all relevant factors which affect the balance.All of these requirements include the latest standard for balancing DIN69888.

\section{Conclusion}

The conclusion can be summarized as following points.

- Previous findings showing that as speeds on machine tools are increased, centrifugal force from unbalance becomes a damaging factor.

- Unbalance can be of three basic types: static, couple and dynamic.

- The tool, toolholder, and retention knob should be balanced as an assembly.

- Unbalance in Toolholders comes from two sources: Fixed and Variable.

- Toolholders used in high speed machining should be two-plane balanced by the manufacturer to remove the Fixed Sources of unbalance.

- Most Variable Sources of unbalance can be removed by single plane balancing.

- The balance tolerance for a given toolholder can be easily calculated using the ISO 1940 Standards, but the Balance Tolerance.

- ISO has deficiencies. It does not take into account the cyclic stress machine tool and does not reflect the quality requirements of the surface. Deficiencies are regulated in DIN69888.

\section{References}

- Borovan, P. (2008) Požadavkynavyváženírotačníchnástrojových system, Technickýtýdeník ,23, p. 27. ISSN : 0040-1064 
- Commtest (2005) Basic of Machine Unbalance. Commtest Instruments Ltd. Available at http://commtest.com/support/category:frequently-asked-questions/imbalance-will-not-reduce/

- Dingding, Z.; Ping, C. (2012) Permanent Calibration in High Frequency Ratio (>0.3) Dynamic Unbalance Measurement, Procedia Engineering, 29, p. 4278-4281. ISSN : 1877-7058

- Grim, G.K.; Haidler, J.W.; Mitchell, B.J. (2008)The Basic of Balancing, Balance Technology Inc.

- Haimer (2009) Manual Haimer Tool Dynamic

- ISO 1940/1 (2003) Mechanical vibration. Balance quality requirements for rotors in a constant (rigid) state.

- Kováč, M.; Zvončan, M.; Peterka, J. (2011) Influence of tool balancing on machined surface quality in High Speed Machining, Journal of Production Engineering, vol. 15 (1), p. 11-14. ISSN : 1863-7353

- Layne, M.H. (2007) Detecting and Correcting Unbalance in Toolholders for High-Speed Machining, American Hofmann Corporation - Balancing News Letter

- McMillan, R.B. (2004) Rotating Machinery - Practical Solutions to Unbalance and Misalignment, The Fairmont Press, Lilburn, ISBN 0-88173-466-7

- Moak, N. (2001) Balancing Act ,Cutting Tool Engineering, vol. 53 (10). ISSN : 0011-4189

- SandvikCoromant (2012) Balance of tool holders. Catalogue . Available at http://www2.coromant.sandvik.com/coromant/pdf/metalworking_products_061/tech_g_2.pdf.

Seco (2012) Tooling 2012.Seco Catalogue, p. 19-20 